\title{
Efficient second-harmonic generation using a semiconductor tapered amplifier in a coupled ring-resonator geometry
}

\author{
Danilo Skoczowsky, ${ }^{1, *}$ Andreas Jechow, ${ }^{1}$ Ralf Menzel, ${ }^{1}$ Katrin Paschke, ${ }^{2}$ and Götz Erbert ${ }^{2}$ \\ ${ }^{1}$ Universität Potsdam, Institut für Physik und Astronomie, Lehrstuhl für Photonik, Karl-Liebknecht-Straße 24-25, \\ 14476 Potsdam, Germany \\ ${ }^{2}$ Ferdinand-Braun-Institut für Höchstfrequenztechnik, Gustav-Kirchhoff-Straße 4, 12489 Berlin, Germany \\ *Corresponding author: danilo.skoczowsky@uni-potsdam.de
}

Received September 30, 2009; revised December 2, 2009; accepted December 3, 2009; posted December 10, 2009 (Doc. ID 117860); published January 15, 2010

A new approach for efficient second-harmonic generation using diode lasers is presented. The experimental setup is based on a tapered amplifier operated in a ring resonator that is coupled to a miniaturized enhancement ring resonator containing a periodically poled lithium niobate crystal. Frequency locking of the diode laser emission to the resonance frequency of the enhancement cavity is realized purely optically, resulting in stable, single-frequency operation. Blue light at $488 \mathrm{~nm}$ with an output power of $310 \mathrm{~mW}$ is generated with an optical-to-optical conversion efficiency of $18 \%$. (C) 2010 Optical Society of America

OCIS codes: $190.2620,140.3325,140.3560,140.3515,140.3410,140.5960$.

Visible-light generation by second-harmonic generation (SHG) of diode laser radiation using enhancement cavities with a nonlinear crystal is a wellestablished technique. The major problem is to lock the laser emission to the resonance frequency of the enhancement cavity or vice versa. The two common approaches are electronic or optical locking. In electronic locking, an error signal proportional to the frequency detuning is generated [1-3]. This signal can be applied, e.g., to the driving current of the diode laser [1] or to a piezo-controlled mirror to adjust the optical length of the enhancement or reference cavity to tune both resonators to a common resonance. In optical locking, light is injected into the laser to force the laser emission to the resonance frequency of the enhancement cavity without further active stabilization [4]. Therefore, the enhancement cavity itself is used to filter out the appropriate frequency [5]. To improve the stability of the laser output, optical locking can be combined with electronic locking [6-8]. Wigley et al. [9] proposed an optical locking setup where a ring resonator with a laser diode as gain element is coupled to a standing-wave resonator. Frequency selection is realized by the standing-wave resonator and a dielectric spike filter. An alternative method for generation of $488 \mathrm{~nm}$ radiation in a passively stable system is based on sum-frequency generation of a tapered diode laser and a solid-state laser [10].

In our approach we propose a new laser system based on two passively coupled ring resonators. The master ring resonator has a tapered amplifier (TA) as the gain element, while the enhancement ring resonator contains a periodically poled lithium niobate (PPLN) crystal for efficient SHG. The enhancement ring resonator is set up as a miniaturized device and is singly resonant for the IR light. Single-frequency operation of the coupled laser system is provided by two frequency-selective elements, the SHG ring resonator and an additional grating.
The experimental setup is shown in Fig. 1. The output beam of the TA $(\lambda=976 \mathrm{~nm})$ is collimated with an aspherical lens (L1: $f=8 \mathrm{~mm}, \mathrm{NA}=0.5)$ and an additional cylindrical lens (L2: $f=150 \mathrm{~mm}$ ) for the slow axis direction. In this way the beam is symmetrized and nearly free of astigmatism. An optical diode (OD) with $40 \mathrm{~dB}$ of isolation protects the amplifier from damage due to backreflected light and forces unidirectional operation of the amplifier. With a spherical lens (L3: $f=50 \mathrm{~mm}$ ) the beam is focused into the SHG ring resonator, which consists of four mirrors (M1M4), the PPLN crystal and two gradient index (GRIN) lenses.

The beam waists of the focus are determined to $w_{0, \text { slow }}=21 \mu \mathrm{m}$ and $w_{0, \text { fast }}=22 \mu \mathrm{m}$ for the slow and fast axis, respectively, with a remaining astigmatism of $0.2 \mathrm{~mm}$. A part of the IR light is transmitted via

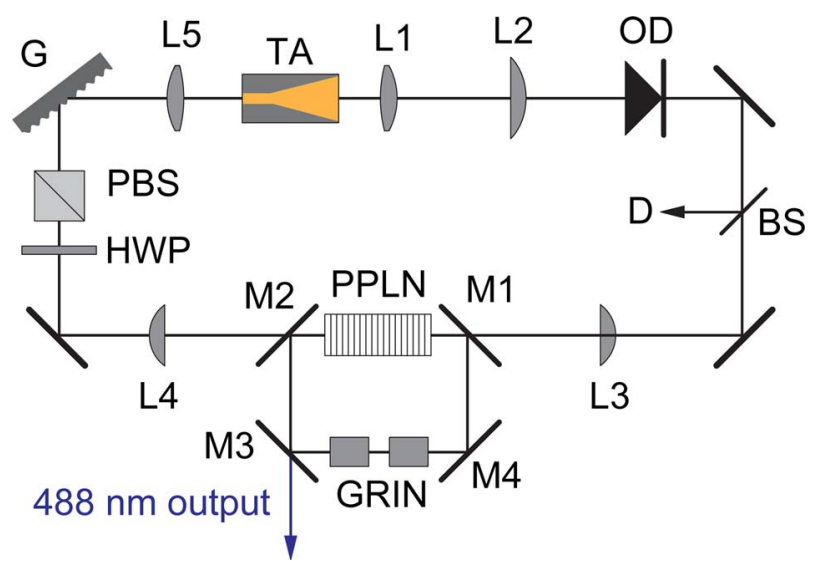

Fig. 1. (Color online) Experimental setup of the coupled resonators with a TA and a miniaturized ring: L1, aspherical lens; L2, cylindrical lens; OD, optical diode; BS, beam splitter; D, diagnostics; L3, spherical lens; mirrors M1-M4; PPLN, periodically poled lithium niobate crystal; GRIN, gradient index lenses; L4, spherical lens; HWP, halfwaveplate; PBS, polarizing beam splitter; G, holographic diffraction grating; L5, aspherical lens. 
mirror M2 and collimated with a spherical lens (L4: $f=125 \mathrm{~mm}$ ). This light is fed back to the rear facet of the TA via a holographic diffraction grating (G: $g$ $=1800 \mathrm{~mm}^{-1}$ ) and an aspherical lens (L5: $f=11 \mathrm{~mm}$, $\mathrm{NA}=0.3$ ). To operate the TA in an appropriate regime, a variable attenuator is used to adjust the amount of feedback light. The attenuator is formed by a rotary $\lambda / 2$ waveplate and a polarizing beam splitter.

The TA is designed with separate contacts for the $1 \mathrm{~mm}$ long ridge and the $3 \mathrm{~mm}$ long tapered section, and therefore the amplifier is mounted $p$-side up. Both facetes are antireflection (AR) coated with a reflectivity of $R<10^{-4}$. The waveguide core in the direction of the fast axis is built as a so-called asymmetric super-large optical-cavity (aSLOC) [11]. This reduces the vertical far field angle as well as the optical power density allowing operation at higher maximum optical output power.

To characterize the TA, a Littrow-type external resonator is used with the same holographic diffraction grating. At injection currents of $150 \mathrm{~mA}$ for the ridge and $3000 \mathrm{~mA}$ for the tapered section, an optical output power of $1770 \mathrm{~mW}$ is measured. The laser emission is tunable from $950 \mathrm{~nm}$ to $990 \mathrm{~nm}$ with a gain maximum of $974 \mathrm{~nm}$ at $T=20.00^{\circ} \mathrm{C}$ and a corresponding linewidth $<50 \mathrm{pm}$. The corresponding beam propagation factors are measured to be $M_{\text {fast }}^{2}$ $=1.4$ and $M_{\text {slow }}^{2}=1.7$ using a scanning slit technique and the second-order moments to determine the beam-waist radii (modified BeamScope P5, DataRay, Inc.).

The PPLN bulk crystal $(l=10 \mathrm{~mm})$ is manufactured by HC Photonics. It is made of congruent melt with $5 \% \mathrm{MgO}$ doping. The poling period is $5.36 \mu \mathrm{m}$ for optimal SHG of $976 \mathrm{~nm}$ at a temperature of $50^{\circ} \mathrm{C}$. A conversion efficiency of $2.7 \% / \mathrm{W}$ is measured in a single-pass setup. Both facets of the crystal are AR coated for the fundamental and second-harmonic wave with a residual reflectivity of each facet of $R_{976 \mathrm{~nm}}<0.5 \%$ and $R_{488 \mathrm{~nm}}<1.0 \%$, respectively.

All parts of the SHG ring resonator are mounted on a glass substrate with a size of $19.5 \mathrm{~mm} \times 8.5 \mathrm{~mm}$, where the PPLN crystal is the size-limiting element. Resonator stability is realized by two GRIN lenses $\left(f_{1}=f_{2}=6.6 \mathrm{~mm}\right)$ that are placed symmetrically to the crystal. The lenses are AR coated for the fundamental wave with a residual reflectivity of each surface of $R<0.2 \%$. By variation of the distance between the two lenses, the beam waist can be adjusted and mode matching can be optimized. The mirror reflectivities are calculated based on input power, efficiency of the crystal, and resonator losses due to nonperfect $A R$ coatings and absorption [12]. Impedance matching is one of the most important aims that can be realized with a reflectivity of the incoupling mirror M1 of $R$ $=0.86$. It turned out that the impedance-matching condition is almost fulfilled for a wide range of cavity losses and reflectivities of the outcoupling mirror M2, and thus the reflectivity of mirror M2 can be chosen without affecting this aim. For sufficient IR light transmission the reflectivity of mirror M2 is chosen to $R=0.96$. Mirrors M3 and M4 are highly reflecting for the fundamental. The blue light is coupled out via mirror M3. The finesse of the SHG ring resonator is estimated from the reflectivities of the mirrors and resonator losses to be approximately 20 including the losses due to SHG. The optical path length of the SHG ring resonator equals $50 \mathrm{~mm}$, which corresponds to a free spectral range (FSR) of $6 \mathrm{GHz}$.

To achieve stable operation, the miniaturized ring resonator is isolated in a Teflon housing and temperature stabilized to $27.10 \pm 0.01^{\circ} \mathrm{C}$. The temperature of the tapered amplifier is set to $20.00 \pm 0.01^{\circ} \mathrm{C}$. At injection currents of $150 \mathrm{~mA}$ for the ridge and $2500 \mathrm{~mA}$ for the tapered section, the output power of the TA is measured to be $1680 \mathrm{~mW}$. The power incident at the SHG ring is $1470 \mathrm{~mW}$ from which $410 \mathrm{~mW}$ is reflected. We assume that this is due to spatial mode mismatch because of the spatial emission properties of the TA with $M^{2}>1.4$ and the fundamental mode operation of the SHG ring. Furthermore, the experimentally determined finesse was 10 . We assume that this reduction by a factor of two is caused by a mode mismatch between the incident light and the SHG resonator mode. Over a period of $10 \mathrm{~min}$ a blue output power of $310 \mathrm{~mW}$ is observed with fluctuations below $1 \%$. The spectrum of the IR light is measured with an optical spectrum analyzer (OSA); see Fig. 2. No side modes are observed, and the spectral emission bandwidth of $50 \mathrm{pm}$ in this measurement is given by the resolution limit of the OSA. The highest conversion efficiency at the chosen temperature of the PPLN crystal is observed at an IR emission wavelength of $975.2 \mathrm{~nm}$.

Single-frequency operation of the coupled resonators is proved by an additional spectral measurement with a scanning Fabry-Perot interferometer (FPI) of type FPI 100 by Toptica Photonics $(\mathrm{FSR}=4 \mathrm{GHz}, \mathcal{F}$ $>400$ ). The two lines in Fig. 2 are separated by the FSR of the scanning FPI, and the linewidth of the IR light is determined to be $50 \mathrm{MHz}$ (sweep time $15 \mathrm{~ms}$ ); see the inset of Fig. 3.

The diffraction grating provides a spectral resolution of $15 \mathrm{GHz}$, which is realized by nearly full illumination of the 15-mm-long grating. By tilting the grating it is possible to tune the IR laser emission in steps of the FSR of the SHG ring resonator. Thus,

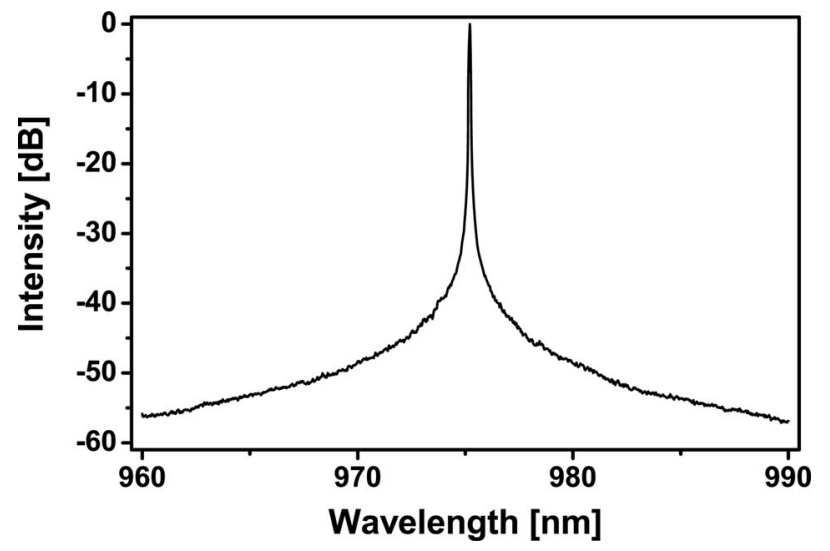

Fig. 2. Spectrum of the IR light measured with an OSA (resolution limit $50 \mathrm{pm}$ ). 


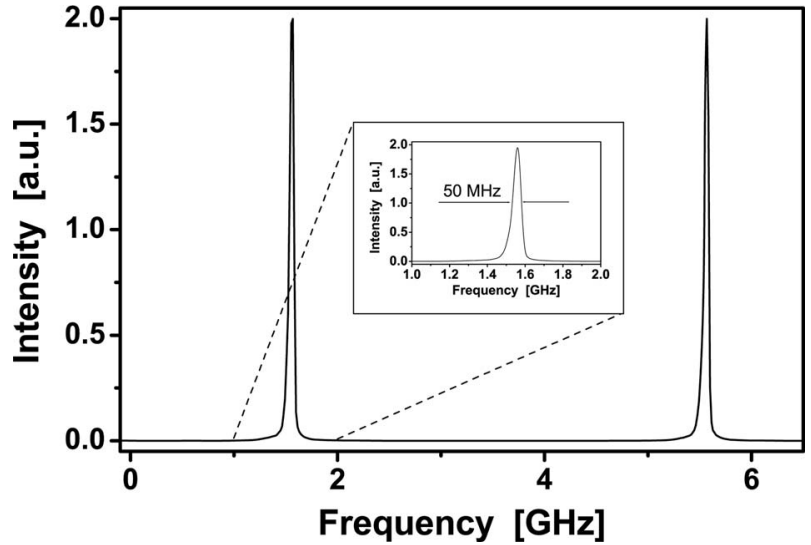

Fig. 3. Spectrum of the IR light measured with a scanning Fabry-Perot interferometer and a zoom of the emission line (inset) showing single-frequency operation of the coupled resonators (sweep time $15 \mathrm{~ms}$ ).

when the temperature of the crystal is changed simultaneously, the blue laser emission can be tuned in steps of $12 \mathrm{GHz}$. The tuning range of the blue light is limited by the used crystal oven having an upper temperature limit of $30^{\circ} \mathrm{C}$. However, wavelength tuning of $1.5 \mathrm{~nm}$ was demonstrated in a single-pass setup using the same PPLN crystal [13].

The blue output beam is nearly diffraction limited with beam propagation factors of $M_{\text {fast }}^{2}=1.05$ and $M_{\text {slow }}^{2}=1.07$ for the fast and slow axis, respectively. The SHG ring resonator acts as an efficient mode filter with fundamental mode oscillation only.

In summary, a diode-laser-based system of two coupled ring resonators for efficient SHG is presented. A tapered amplifier in a low-finesse ring resonator is used to drive SHG in a second ring resonator with nonlinear crystal. Because of the high output power of the TA and the high efficiency of the PPLN crystal, the SHG ring resonator is designed with a relatively low finesse. The adjustment of the laser system is uncritical compared to common high finesse setups. Frequency locking of both resonators is realized purely optically. Stable output power of $310 \mathrm{~mW}$ of blue light at $488 \mathrm{~nm}$ is observed. The emitted blue light is single frequency with a linewidth of $50 \mathrm{MHz}$ and nearly diffraction limited with $M^{2}<1.1$ for both axes. This scheme can be adapted to other diode lasers to generate its second harmonic in an efficient way.

\section{References}

1. W. J. Kozlovsky, W. Lenth, E. E. Latta, A. Moser, and G. L. Bona, Appl. Phys. Lett. 56, 2291 (1990).

2. T. W. Hänsch and B. Couillaud, Opt. Commun. 35, 441 (1980).

3. R. W. P. Drever, J. L. Hall, F. V. Kowalski, J. Hough, G. M. Ford, A. J. Munley, and H. Ward, Appl. Phys. B 31, 97 (1983).

4. B. Dahmani, L. Hollberg, and R. Drullinger, Opt. Lett. 12, 876 (1987).

5. G. J. Dixon, C. E. Tanner, and C. E. Wieman, Opt. Lett. 14, 731 (1989).

6. A. Hemmerich, D. H. McIntyre, C. Zimmermann, and T. W. Hänsch, Opt. Lett. 15, 372 (1990).

7. W. J. Kozlovsky, W. P. Risk, W. Lenth, B. G. Kim, G. L. Bona, H. Jaeckel, and D. J. Webb, Appl. Phys. Lett. 65, 525 (1994).

8. D. Woll, B. Beier, K.-J. Boller, R. Wallenstein, M. Hagberg, and S. O’Brien, Opt. Lett. 24, 691 (1999).

9. P. G. Wigley, Q. Zhang, E. Miesak, and G. J. Dixon, Opt. Lett. 20, 2496 (1995).

10. E. Karamehmedović, C. Pedersen, M. T. Andersen, and P. Tidemand-Lichtenberg, Opt. Express 15, 12240 (2007).

11. H. Wenzel, F. Bugge, M. Dallmer, F. Dittmar, J. Fricke, K. H. Hasler, and G. Erbert, IEEE Photon. Technol. Lett. 20, 214 (2008).

12. D. Skoczowsky, A. Jechow, H. Stürmer, T. Poßner, S. Stry, J. Sacher, and R. Menzel, Appl. Phys. B 10.1007/ s00340-009-3802-7 (2009).

13. A. Jechow, V. Raab, and R. Menzel, Appl. Opt. 46, 943 (2007). 